\title{
Combined Impact of Pre-sensitization and Delayed Graft Function on Allograft Rejection in Deceased Donor Kidney Transplantation: Nationwide Cohort Study
}

Hanbi Lee

Seoul St. Mary's Hospital

Yohan Park

Seoul St. Mary's Hospital

Tae Hyun Ban

Eunpyeong St. Mary's hospital

Sang Heon Song

Pusan National University Hospital

\section{Seung Hwan Song}

Ewha Womans University Medical Center

Jaeseok Yang

Seoul National University Hospital

\section{Curie Ahn}

Seoul National University Hospital

Chul Woo Yang

Seoul St. Mary's Hospital

Byung Ha Chung ( $\nabla$ chungbh@catholic.ac.kr)

Seoul St. Mary's Hospital

\section{Research Article}

Keywords: delayed graft function (DGF), deceased donor kidney transplantation (DDKT), post-transplant complications

Posted Date: February 25th, 2021

DOl: https://doi.org/10.21203/rs.3.rs-227446/v1

License: (9) This work is licensed under a Creative Commons Attribution 4.0 International License.

Read Full License 
Version of Record: A version of this preprint was published at Scientific Reports on August 9th, 2021. See the published version at https://doi.org/10.1038/s41598-021-95327-6. 


\section{Combined Impact of Pre-sensitization and Delayed Graft Function on Allograft}

\section{Rejection in Deceased Donor Kidney Transplantation: Nationwide Cohort Study}

Hanbi Lee ${ }^{1,+}$, Yohan Park ${ }^{1,+}$, Tae Hyun Ban ${ }^{2}$, Sang Heon Song ${ }^{3}$, Seung Hwan Song ${ }^{4}$, Jaeseok Yang $^{5}$, Curie Ahn ${ }^{5}$, Chul Woo Yang ${ }^{1}$, Byung Ha Chung ${ }^{1, *} \&$ the Korean Organ Transplantation Registry Study Group

${ }^{1}$ Division of Nephrology, Department of Internal Medicine, Seoul St. Mary’s Hospital. ${ }^{2}$ Division of Nephrology, Department of Internal Medicine, Eunpyeong St. Mary's Hospital. ${ }^{3}$ Organ Transplantation Center and Department of Internal Medicine, Pusan National University Hospital. ${ }^{4}$ Department of Surgery, Ewha Womans University Medical Center. ${ }^{5}$ Department of Nephrology, Seoul National University Hospital.

+ These authors contributed equally to this work.

$\$$ A comprehensive list of the Korean Organ Transplantation Registry Study Group members appears at the end of the paper.

* email: chungbh@ catholic.ac.kr 


\begin{abstract}
The aim of this study is to investigate whether or not delayed graft function (DGF) and pretransplant sensitization has a synergistic adverse effect on allograft outcome after deceased donor kidney transplantation (DDKT) using the Korean Organ Transplantation Registry (KOTRY) database, the nationwide prospective cohort. The study included 1,370 cases between May 2014 and June 2019. The cases were divided into 4 subgroups according to presensitization and the development of DGF post-transplant (non-pre-sensitized-DGF()$(n=1100)$, non-pre-sensitized-DGF $(+)(n=133)$, pre-sensitized-DGF(-) $(n=116)$, and presensitized-DGF $(+)(n=21))$. We compared the incidence of biopsy-proven allograft rejection (BPAR), time-related change in allograft function, allograft or patient survival, and posttransplant complications across 4 subgroups. The incidence of overall BPAR and acute antibody-mediated rejection (ABMR) was significantly higher in the pre-sensitized-DGF(+) subgroup than in other 3 subgroups. In addition, multivariable cox regression analysis demonstrated that pre-sensitization combined with DGF is an independent risk factor for both overall BPAR (hazard ratio 3.253, $\mathrm{p}=0.005$ ) and acute ABMR (hazard ratio 7.589, $\mathrm{p}<$ 0.001). Moreover, DGF and pre-sensitization showed significant interaction. Pre-sensitization combined with DGF did not show significant impact on allograft function, and allograft or patient survival. In conclusion, pre-sensitization and DGF had a synergistic adverse impact on allograft rejection after DDKT.
\end{abstract}




\section{Introduction}

Delayed graft function (DGF) is a manifestation of acute kidney injury (AKI), which is more prevalent in deceased donor kidney transplantation (DDKT). The definition of DGF varies according to the study; however, it is mostly based on the use of dialysis within 1 week from transplant ${ }^{1-3}$. The mechanism underlying the development of DGF still needs to be unveiled, but it is suggested that post-ischemic acute tubular necrosis resulting from ischemia and reperfusion injury (IRI) developing during deceased donor management or recovery of organs, and calcineurin inhibitor (CNI) toxicity may be the major contributors ${ }^{4}$. The activation of adaptive immune system induced by DGF also increases the risk of allograft rejection.

Meanwhile, it is well known that the presence of preexisting donor-specific anti-human leukocyte antigen antibody (HLA-DSA), so-called "pre-sensitized state" is an important obstacle preventing successful kidney transplantation (KT) ${ }^{5-9}$. In such patients, HLA-DSA can increase the risk of acute or chronic antibody-mediated rejection (ABMR) resulting in worse allograft outcomes ${ }^{10,11}$. In the setting of DDKT, DGF combined with subclinical rejection resulted in far worse allograft outcomes. In addition, the detrimental impact of DGF on allograft was enhanced by the presence of pre-transplant HLA-DSA in DDKT ${ }^{12}$.

Based on the above background, it is possible that DGF in patients with pre-sensitization has a synergistic adverse impact on the allograft outcomes. However, it has yet to be fully investigated and only a single center study is available ${ }^{12}$. In this regard, the aim of this study is to investigate the combined impact of DGF and pre-sensitization on the development of 
allograft rejection using the well-established nationwide prospective cohort, Korean Organ Transplantation Registry (KOTRY).

\section{Results}

\section{Baseline clinical and immunological patient characteristics}

DGF developed in $11.2 \%(154 / 1370)$ out of the total DDKT recipients. Between presensitized and non-pre-sensitized subgroups, no difference was detected in the frequency of DGF $(10.8 \%$ vs. $15.3 \%, p=0.110)$. Table 1 describes baseline characteristics of the donor and recipients of four subgroups. Baseline estimated glomerular filtration rate (eGFR) was significantly lower in donors of DGF (+) subgroups irrespective of pre-sensitization. Cold ischemic time showed a longer tendency in DGF (+) subgroups irrespective of presensitization without statistical significance. However, donor age, gender, body mass index (BMI), underlying disease including DM or hypertension (HTN) and the proportion of donors after cardiac death (DCD) or donors after brain death (DBD) did not differ significantly across 4 subgroups. Among recipient factors, there was a significantly longer dialysis vintage and also additional number of female patients in both pre-sensitized subgroups than in nonpre-sensitized subgroups. In addition, a previous KT history and the proportion of antithymocyte globulin (ATG) use as induction therapy were higher in the pre-sensitized subgroups than in the non-pre-sensitized subgroups. The proportion of DM as the primary renal disease was lower in the pre-sensitized subgroups than in the non-pre-sensitized subgroups. A significantly higher proportion of patients undergoing hemodialysis as the dialysis modality prior to KT were selected from the non-pre-sensitized-DGF(+) subgroup, when compared with the non-pre-sensitized-DGF(-) subgroup. Although the majority of 
patients received tacrolimus as the main immunosuppressant, more patients in $\mathrm{DGF}(+)$ subgroups received sirolimus compared with DGF(-) subgroups.

\section{Comparison of overall biopsy-proven allograft rejection (BPAR) and acute ABMR}

The incidence of overall BPAR was the highest in the pre-sensitized-DGF(+) subgroup $(33.3 \%, 7 / 21)$. The incidence rate of acute ABMR was higher in the pre-sensitized-DGF(+) subgroup $(23.8 \%, 5 / 21)$ than in other 3 subgroups. The incidence of chronic ABMR was higher in pre-sensitized subgroups compared to non-pre-sensitized subgroups. In contrast, acute and chronic T-cell mediated rejection (TCMR) rate showed no statistically significant difference across four subgroups (Table 2).

The Kaplan-Meier curve showed that cumulative overall BPAR rate was highest in the pre-sensitized-DGF(+) subgroup (log rank; $p=0.001$ vs. non-pre-sensitized-DGF(-), $p=0.014$ vs. non-pre-sensitized-DGF(+), $\mathrm{p}=0.009$ vs. pre-sensitized-DGF(-)) (Figure 2a). Similarly, cumulative acute ABMR rate was highest in the pre-sensitized-DGF(+) subgroup (log rank; $\mathrm{p}<0.001$ vs. non-pre-sensitized-DGF(-), $\mathrm{p}=0.001$ vs. non-pre-sensitized-DGF( + ), $\mathrm{p}=0.009$ vs. pre-sensitized-DGF(-)) (Figure 2b).

\section{Risk factors of overall BPAR and acute ABMR}

In the adjusted multivariable regression model, the hazard ratio (HR) of overall BPAR was the highest in pre-sensitized-DGF (+) subgroup compared to the reference of non-presensitized-DGF(-) subgroup (HR 3.253, 95\% CI: 1.423-7.434, p=0.005). A significant interaction was found between DGF and pre-sensitization $(\mathrm{p}=0.006)$ (Table 3a). Combination of pre-sensitization and DGF was also an independent risk factor for acute ABMR (HR 7.589 
vs. non-pre-sensitized-DGF(-) subgroup, 95\% CI: 2.644-21.777, p<0.001). Moreover, DGF and pre-sensitization showed statistically significant interaction $(\mathrm{p}<0.001)$ (Table $3 \mathrm{~b})$.

\section{Comparison of the change in allograft function and death-censored allograft survival}

At the end of the 3-year follow-up, allograft function measured by eGFR using chronic kidney disease-epidemiology collaboration (CKD-EPI) equation was the lowest in non-presensitized-DGF(+) subgroup. The change in time-related allograft function of non-presensitized-DGF(+) subgroup was significantly different from that of other subgroups in linear mixed model (Figure 3) ( $\mathrm{p}<0.001$ vs. non-pre-sensitized-DGF(-), $\mathrm{p}<0.001$ vs. pre-sensitizedDGF(-), $\mathrm{p}=0.034$ vs. pre-sensitized-DGF(+)).

Totally, 52 cases of allograft failure developed during the follow-up period. The main factor contributing to allograft loss was rejection $(17 / 52,32.7 \%)$, followed by primary graft failure $(11 / 52,21.2 \%)$. In non-pre-sensitized-DGF(-) subgroup, rejection was the main cause of allograft loss $(13 / 33,39.4 \%)$, followed by glomerulonephritis $(4 / 33,12.1 \%)$, primary graft failure $(3 / 33,9.1 \%)$. In non-pre-sensitized-DGF(+) subgroup, the main cause of allograft loss was primary graft failure $(6 / 14,42.9 \%)$, followed by rejection $(3 / 14,21.4 \%)$. In presensitized-DGF(-) subgroup, both rejection and postoperative complications accounted for $33.3 \%$, respectively. In pre-sensitized-DGF(+) subgroup, primary graft failure was the only reason for allograft loss. The Kaplan-Meier curve showed lowest death-censored allograft survival in the non-pre-sensitized-DGF(+) subgroup (log rank; $\mathrm{p}<0.001$ vs. non-presensitized-DGF(-), $\mathrm{p}=0.013$ vs. pre-sensitized-DGF(-)); however, there was no significant difference compared with pre-sensitized-DGF(+) subgroup (log rank $\mathrm{p}=0.958)$ (Figure 4). 


\section{Comparison of patient survival and post-transplant complications}

A total of $57(4.1 \%)$ patients died in our cohort due to cardiovascular disease in 9 cases, infection in 27 , malignancy in 4 , others (liver disease, cerebral infarction, acute CNI toxicity, gastrointestinal bleeding, and acute rejection etc.) in 12, and unknown etiology in 5 cases. In each subgroup, 37 (3.4\%) died in the non-pre-sensitized-DGF(-) subgroup, 16 (12.0\%) in the pre-sensitized-DGF(+) subgroup, 4 (3.4\%) in the pre-sensitized-DGF(-) subgroup, and none $(0.0 \%)$ in the pre-sensitized-DGF(+) subgroup. The total death rate was the highest in the non-pre-sensitized-DGF(+) subgroup $(\mathrm{p}=0.001)$ (Table 4a).

There was no significant difference in development of BK virus-associated nephropathy (BKVAN), cerebrovascular disease, infectious complications and malignancy across the four subgroups (Table 4b). In regard to cardiovascular disease, the pre-sensitized-DGF(+) subgroup showed a lower incidence compared to non-pre-sensitized-DGF(+) subgroup $(p=0.026)$. Although the overall infection rate did not differ across 4 subgroups $(\mathrm{p}=0.316)$, the incidence of Pneumocystis jirovecii pneumonia (PJP) infection was the highest in the presensitized-DGF(+) subgroup $(1 / 21,7.1 \%, \mathrm{p}=0.046)$.

\section{Discussion}

Pre-sensitization to HLA is a well-known pre-transplant factor, which can increase the risk for allograft rejection and allograft failure. Meanwhile, DGF is a well-known post-transplant factor, which also induces adverse allograft outcomes. This study demonstrated that the combination of post-transplant factor (DGF) and pre-transplant risk factor (pre-sensitization) had a synergistic adverse effect on allograft outcomes, especially higher incidence of allograft rejection. 
First, we compared baseline characteristics of donors and recipients across 4 clinical subgroups. In terms of donor factors, baseline renal function was significantly lower in patients who showed DGF, which was consistent with previous studies, which reported that low baseline kidney function is a risk factor for DGF ${ }^{13}$. In contrast, there was no significant difference in the frequency of DGF between pre-sensitized and non-pre-sensitized subgroups, which suggests that pre-sensitization may not have a significant effect on the development of DGF. Among recipient factors, the dialysis was significantly prolonged in pre-sensitized subgroups, which suggested that sensitized subjects need longer wait time for DDKT allocation ${ }^{14-16}$. As expected, the proportion of female recipients was higher in both presensitized subgroups ${ }^{15}$ and the proportion of recipients with previous KT history was higher and tended to be high in both pre-sensitized subgroups than in non-pre-sensitized subgroups. In addition, although a majority of patients received primary maintenance immunosuppression with tacrolimus, a significantly higher number of patients received sirolimus in the non-pre-sensitized-DGF(+) subgroup. This finding suggested that physicians decided a switch from CNI to mammalian target of rapamycin (mTOR) inhibitor, given that CNI may contribute to delayed recovery of allograft function ${ }^{17}$.

Second, we compared the incidence of overall BPAR according to pre-sensitization or the development of DGF. As a result, the incidence of overall BPAR was the highest in the presensitized-DGF(+) subgroup and was mainly attributed to the increase in acute ABMR rather than acute and chronic TCMR as expected. Interestingly, pre-sensitization and DGF showed significant interaction with each other, which suggests their synergistic impact on the 
development of acute ABMR. This finding can be explained by two factors. First, DGF per se can increase the immunogenicity of allograft, and thereby increase the vulnerability to immune reaction of pre-formed HLA-DSA. Indeed, IRI in DGF can up-regulate the major histocompatibility complex (MHC) class I and II antigens, and enhance the expression of adhesion and costimulatory molecules of allograft tissue ${ }^{18-21}$. Moreover, the IRI induces ligands of toll-like receptors (TLRs) and activate cells of the innate immune system, inducing activation and maturation of dendritic cells, followed by adaptive immune response ${ }^{21}$. Indeed, the previous studies demonstrated that DGF is associated with an increased risk of allograft loss and acute rejection ${ }^{22,23}$. Second, the conversion of CNI to mTOR inhibitor was more frequently detected in patients who suffered from DGF in this study, perhaps because CNI might be considered as a contributor to DGF. Lower suppressive potency of mTOR inhibitor for humoral immunity in comparison with tacrolimus is another possible cause of higher rate of acute ABMR in pre-sensitized-DGF(+) subgroup ${ }^{24}$.

Surprisingly, pre-sensitization or DGF per se had no significant effect on the development of overall BPAR. The reason is unclear, but it may be attributed to the limited definition of both pre-sensitization and DGF in the study using a nationwide cohort. In case of presensitization, since data of DSA were collected from 2017, the results of HLA-DSA were not available in some recipients. Therefore, in such recipients, we defined sensitization to HLA by the presence of panel reactive antibodies (PRA), together with positive crossmatch test results. Even though this definition is used for "pre-sensitization", we cannot assess the degree of sensitization clearly. In case of DGF, the definition of DGF is varies among previous studies ${ }^{25}$. Indeed, the definition of DGF merely depends on the performance of 
dialysis after KT, and the decision whether or not to perform dialysis can differ according to the transplant centers. In addition, due to the absence of detail data, individualized immunosuppression regimen according to immunologic risk stratification and the serum level of immunosuppressant in each recipient did not be considered in our analysis. Therefore, the aforementioned factors can induce bias that can affect the result of this study.

Interestingly, non-pre-sensitized-DGF(+) group showed worst allograft function at 36 months post-transplant follow-up and worst allograft survival. One of the possible reasons is the baseline status of the corresponding donor kidney (Suppl. Table 1). The donor of this group showed the least renal function at baseline and a relatively higher kidney donor profile index (KDPI) score. In addition, in 5 out of 14 patients with allograft loss in this group, primary non-function was the reason for allograft failure. All of the foregoing findings suggest that the baseline status of donor kidney was the worst in this group, which may result in a higher rate of allograft loss and also sustained low allograft function. In regard to allograft survival, the impact of the baseline kidney function can be more significant than allograft rejection during the limited follow-up duration. Hence, the allograft survival was not the lowest in the pre-sensitized-DGF(+) subgroup in spite of the highest rate of BPAR.

Lastly, we compared the post-transplant complications among the 4 subgroups. Non-presensitized-DGF(+) subgroup showed the higher patient death rate. However, only 57 cases out of 1,370 kidney transplantation recipients (KTRs) were found and there was no patient death in the pre-sensitized-DGF(+) subgroup. Therefore, longer observations may be required to arrive at any conclusion. Compared with the post-transplant infection, no difference was 
detected across 4 subgroups in most infection types, except that the rate of PJP infection was the highest in the pre-sensitized-DGF(+) subgroup. However, there was only 1 case of PJP infection, hence further investigation may be required to clarify this issue as well ${ }^{26}$.

This study has some limitations. First, this nationwide registry analysis reflects similar limitations found in similar large registry analyses as shown in our previous studies ${ }^{27}$. While patient numbers are enhanced, important details for the endpoints are missing, thereby reducing the clinical utility of the findings. For example, the HLA-DSA was not available for analysis in some patients, and we cannot use the class and the strength of DSA in the analysis, which has been reported as an important risk factor for allograft rejection and failure ${ }^{6,10,28-30}$. Second, the follow-up duration of this registry is limited as mentioned previously. Therefore, traditional risk factors for allograft failure such as DGF and pre-sensitization did not significantly affect allograft outcome. Third, we could not determine the specified protocols at each center in DDKT for highly sensitized recipients. Despite pre-transplant desensitization was performed in 37 recipients, including those whom with positive B-cell crossmatch, no data were available on the protocol. Some centers used rituximab to treat such patients, and others did not, but unfortunately, it was not considered in this analysis. Nevertheless, our study is the first prospective, multi-centered cohort study to investigate the association of DGF and pre-sensitization in allograft outcomes.

In conclusion, we have shown that combination of DGF and pre-sensitization to HLA had a detrimental impact on allograft outcome in terms of rejection. Therefore, we suggest that more careful monitoring or surveillance of allograft rejection is required. Further, we need to 
use more intensified immunosuppression protocol to prevent allograft rejection when DGF occurred in DDKT with pre-sensitization.

\section{Methods}

\section{Study population}

We analysed KOTRY data from the Korean Society for Transplantation ${ }^{31}$, compiling data from 30 kidney transplantation centers in Korea ${ }^{32}$. The KOTRY data includes 1,945 DDKT cases between May 2014 and June 2019, from which we excluded 575 DDKT recipients with unavailable data regarding PRA, HLA-DSA, crossmatch tests or DGF development. Therefore, we included 1,370 DDKT recipients in the present investigation and classified the patients into four subgroups according to the pre-sensitization and the development of DGF post-transplant: non-pre-sensitized-DGF(-) $(n=1100)$, non-pre-sensitized-DGF $(+) \quad(n=133)$, pre-sensitized-DGF(-) $(n=116)$, pre-sensitized-DGF $(+) \quad(n=21)$ (Figure 1). The median follow-up period of this study was 38.0 (interquartile range: 25.0-50.8) months.

We defined pre-sensitization to HLA by the presence of (i) HLA-DSA (by Luminex single antigen assay) or (ii) PRA (by solid-phase HLA antibody screening), combined with positive crossmatch test results. HLA-DSA data were available in 1,060 recipients $(77.3 \%)$. Therefore, the sensitization to HLA was defined by the detection of HLA-DSA in those patients. In another 310 DDKT recipients for whom HLA-DSA data were not available, we defined sensitization to HLA based on the positive results of PRA and crossmatch test. Among 310 DDKT recipients, 5 recipients were sensitization to HLA with the presence of PRA and positive B-cell crossmatch. DGF was defined as the need for dialysis within 1 week of 
transplantation. The medical records were reviwed after receiving informed consent ${ }^{32}$. This study was performed in accordance with the Declaration of Helsinki and the Declaration of Istanbul. The study was approved by the Institutional Review Board of Seoul St. Mary's Hospital (KC14ONMI0460).

\section{Definition of clinical outcomes}

The clinical outcomes investigated in this study included the incidence of overall BPAR, acute ABMR, time-related changes in allograft function measured as eGFR, death-censored allograft survival rates, and post-transplant complications such as BKVAN, cardiovascular disease, cerebrovascular disease, infection and malignancy. BPAR was diagnosed according to the Banff 2013 classification ${ }^{33}$. Rejection-free allograft survival was defined as the time elapsed from transplantation to the first episode of BPAR. Serum creatinine levels were collected at six months and later at one-year intervals post-transplant. The eGFR for each concordant time was assessed using the CKD-EPI equation ${ }^{34}$. Allograft survival was defined as the time from transplantation to initiation with alternative renal replacement therapy. Cardiovascular disease is defined as cardiovascular death, myocardial infarction, ischemic heart disease with relevant clinical evidence (accompanied by therapeutic intervention or objective findings), new-onset congestive heart failure requiring hospitalization, and arrhythmia. Cerebrovascular disease included non-traumatic hemorrhagic or ischemic brain disease confirmed by computed tomography or magnetic resonance imaging ${ }^{32}$. BKVAN was diagnosed by allograft biopsy. All clinical parameters were compared across the four patient subgroups. 


\section{Statistical method}

All continuous variables were expressed as mean \pm standard deviation. If the variables followed the normal distribution, an analysis of variance (ANOVA) was performed. If the variables showed non-normal distribution, a Kruskal-Wallis test was performed. Tukey's method or Mann-Whitney test was performed as a post-hoc analysis. All categorical variables were compared using the chi-square test or Fisher's exact test and expressed as proportions. Withdrawal-censored allograft rejection rate and death-censored allograft survival rate were evaluated by using the Kaplan-Meier survival analysis and were compared using the log-rank test. The effects of DGF and pre-sensitization, and the interaction between DGF and presensitization on overall BPAR or acute ABMR were analyzed via Cox proportional-hazards regression analysis. Baseline clinical and laboratory parameters showing significant differences ( $\mathrm{p}$ value $<0.1$ ) in univariable analysis or known to affect allograft rejection were fitted into the multivariable model. We selected donor factors (age, cold ischemic time, KDPI) and recipient factors (BMI, mismatch number, prior KT history, PRA $>50 \%$ ) as confounders. Time-related allograft function between subgroups were compared using a linear mixed model. All missing data were censored from the last follow-up date. P values < 0.05 were statistically significant. All statistical analyses were performed using the SPSS $®$ software, version 24 (IBM Corporation, Armonk, NY, USA) and Microsoft Excel 2016. 


\section{References}

1 Agarwal, A., Murdock, P. \& Fridell, J. A. Comparison of histidine-tryptophan ketoglu tarate solution and University of Wisconsin solution in prolonged cold preservation 0 f kidney allografts. Transplantation 81, 480-482, doi:10.1097/01.tp.0000196724.8975 7.79 (2006).

2 Roels, L. et al. Inferior outcome of cadaveric kidneys preserved for more than $24 \mathrm{hr}$ in histidine-tryptophan-ketoglutarate solution. Leuven Collaborative Group for Trans plantation. Transplantation 66, 1660-1664, doi:10.1097/00007890-199812270-00015 (1998).

3 Yarlagadda, S. G. et al. Marked variation in the definition and diagnosis of delayed graft function: a systematic review. Nephrol Dial Transplant 23, 2995-3003, doi:10.1 093/ndt/gfn158 (2008).

4 Schroppel, B. \& Legendre, C. Delayed kidney graft function: from mechanism to tra nslation. Kidney Int 86, 251-258, doi:10.1038/ki.2014.18 (2014).

5 Sethi, S. et al. Desensitization: Overcoming the Immunologic Barriers to Transplantat ion. J Immunol Res, doi:Artn 6804678

10.1155/2017/6804678 (2017).

6 Chung, B. H. et al. Clinical impact of the baseline donor-specific anti-human leuko cyte antigen antibody measured by Luminex single antigen assay in living donor kid ney transplant recipients after desensitization therapy. Transplant International 27, 49 -59, doi:10.1111/tri.12199 (2014).

7 Haririan, A. et al. Positive Cross-Match Living Donor Kidney Transplantation: Longer -Term Outcomes Editorial Comment. J Urology 182, 1138-1138 (2009).

8 Marfo, K., Lu, A., Ling, M. \& Akalin, E. Desensitization Protocols and Their Outcom e. Clin J Am Soc Nephro 6, 922-936, doi:10.2215/Cjn.08140910 (2011).

9 Caro-Oleas, J. L. et al. Clinical relevance of HLA donor-specific antibodies detecte d by single antigen assay in kidney transplantation. Nephrol Dial Transp/ 27, 1231-1 238, doi:10.1093/ndt/gfr429 (2012).

10 Lefaucheur, C. et al. Preexisting donor-specific HLA antibodies predict outcome in kidney transplantation. J Am Soc Nephro/ 21, 1398-1406, doi:10.1681/ASN.2009101 065 (2010).

11 Mohan, S. et al. Donor-specific antibodies adversely affect kidney allograft outcome s. J Am Soc Nephrol 23, 2061-2071, doi:10.1681/ASN.2012070664 (2012).

12 Haller, J. et al. Differential Impact of Delayed Graft Function in Deceased Donor Re nal Transplant Recipients With and Without Donor-specific HLA-antibodies. Transpla ntation 103, e273-e280, doi:10.1097/TP.0000000000002802 (2019).

13 Mannon, R. B. Delayed Graft Function: The AKI of Kidney Transplantation. Nephron 140, 94-98, doi:10.1159/000491558 (2018).

14 Keith, D. S. \& Vranic, G. M. Approach to the Highly Sensitized Kidney Transplant C andidate. Clin J Am Soc Nephrol 11, 684-693, doi:10.2215/CJN.05930615 (2016).

15 Bostock, I. C. et al. Probability of deceased donor kidney transplantation based on \% PRA. Transp/ Immunol 28, 154-158, doi:10.1016/j.trim.2013.05.002 (2013).

16 Jeon, H. J. et al. Outcomes of end-stage renal disease patients on the waiting list for deceased donor kidney transplantation: A single-center study. Kidney Res Clin P ract 38, 116-123, doi:10.23876/j.krcp.18.0068 (2019).

17 Diekmann, F. et al. Sequential quadruple immunosuppression including sirolimus in extended criteria and nonheartbeating donor kidney transplantation. Transplantation 8 
4, 429-432, doi:10.1097/01.tp.0000269610.13590.52 (2007).

de Sandes-Freitas, T. V. et al. Prolonged Delayed Graft Function Is Associated with Inferior Patient and Kidney Allograft Survivals. PLoS One 10, e0144188, doi:10.1371 /journal.pone.0144188 (2015).

19 Shoskes, D. A., Parfrey, N. A. \& Halloran, P. F. Increased major histocompatibility c omplex antigen expression in unilateral ischemic acute tubular necrosis in the mous e. Transplantation 49, 201-207, doi:10.1097/00007890-199001000-00045 (1990).

20 Doshi, M. D., Garg, N., Reese, P. P. \& Parikh, C. R. Recipient risk factors associat ed with delayed graft function: a paired kidney analysis. Transplantation 91, 666-67 1, doi:10.1097/TP.0b013e318209f22b (2011).

21 Land, W. G. The role of postischemic reperfusion injury and other nonantigen-depe ndent inflammatory pathways in transplantation. Transplantation 79, 505-514, doi:10. 1097/01.tp.0000153160.82975.86 (2005).

22 Yarlagadda, S. G., Coca, S. G., Formica, R. N., Jr., Poggio, E. D. \& Parikh, C. R. Association between delayed graft function and allograft and patient survival: a syst ematic review and meta-analysis. Nephrol Dial Transplant 24, 1039-1047, doi:10.10 93/ndt/gfn667 (2009).

23 Butala, N. M., Reese, P. P., Doshi, M. D. \& Parikh, C. R. Is delayed graft function causally associated with long-term outcomes after kidney transplantation? Instrumen tal variable analysis. Transplantation 95, 1008-1014, doi:10.1097/TP.0b013e3182855 544 (2013).

24 Croze, L. E. et al. Conversion to mammalian target of rapamycin inhibitors increase s risk of de novo donor-specific antibodies. Transp/ Int 27, 775-783, doi:10.1111/tr i. 12330 (2014)

25 Mallon, D. H., Summers, D. M., Bradley, J. A. \& Pettigrew, G. J. Defining delayed graft function after renal transplantation: simplest is best. Transplantation 96, 885-8 89, doi:10.1097/TP.0b013e3182a19348 (2013).

26 Lee, S. H. et al. Risk factors for Pneumocystis jirovecii pneumonia (PJP) in kidney $t$ ransplantation recipients. Sci Rep 7, 1571, doi:10.1038/s41598-017-01818-w (2017).

27 Ko, E. J., Yu, J. H., Yang, C. W., Chung, B. H. \& Korean Organ Transplantation Re gistry Study, G. Clinical outcomes of $\mathrm{ABO}-$ and HLA-incompatible kidney transplant ation: a nationwide cohort study. Transp/ Int 30, 1215-1225, doi:10.1111/tri.12979 (2017).

28 Riethmuller, S. et al. Donor-specific antibody levels and three generations of cross matches to predict antibody-mediated rejection in kidney transplantation. Transplant ation 90, 160-167 (2010).

29 Chung, B. H. et al. Impact of the Baseline Anti-A/B Antibody Titer on the Clinical Outcome in ABO-Incompatible Kidney Transplantation. Nephron Clinical Practice 124, 79-88, doi:10.1159/000355855 (2013).

30 Toki, D. et al. Acute antibody-mediated rejection in living ABO-incompatible kidney transplantation: long-term impact and risk factors. Am J Transplant 9, 567-577, do $\mathrm{i}: 10.1111 / \mathrm{j} .1600-6143.2008 .02538 . x$ (2009).

31 Ahn, C. et al. Initial Report of the Korean Organ Transplant Registry: The First Repo rt of National Kidney Transplantation Data. Transp/ P 46, 425-430, doi:10.1016/j.tran sproceed.2013.11.083 (2014).

32 Yang, J. et al. Design and Methods of the Korean Organ Transplantation Registry. $T$ ransplant Direct 3, e191, doi:10.1097/TXD.0000000000000678 (2017).

33 Haas, M. et al. Banff 2013 meeting report: inclusion of c4d-negative antibody-medi ated rejection and antibody-associated arterial lesions. Am J Transplant 14, 272-28 
3, doi:10.1111/ajt.12590 (2014).

34 Levey, A. S. et al. A New Equation to Estimate Glomerular Filtration Rate. Ann Inter $n$ Med 150, 604-612 (2009).

\section{Acknowledgements}

This research was supported by a fund (2014-ER6301-00, 2014-ER6301-01, 2014-ER630102, 2017-ER6301-00, 2017-ER6301-01, 2017-ER6301-02) by Research of Korea Centers for Disease Control and Prevention and Research Fund of Seoul St. Mary's Hospital, The Catholic University of Korea and also supported by a grant from the Korean Health Technology R\&D Project, Ministry of Health \& Welfare, Republic of Korea (HI20C0317).

\section{Authors contributions}

HL: participated in designing study, analyzing and interpreting the data, and writing the paper. YP: participated in analyzing the data, and writing the paper. THB: participated in collecting data. SHS: participated in collecting data. SHS: participated in collecting data. JY: participated in collecting data. CA: participated in collecting data. CWY: participated in collecting data. BHC: participated in designing study, analyzing and interpreting the data, and revising the paper. KOTRY study group: participated in collecting data.

\section{Additional Information}

\section{Data availability}

The datasets generated during and/or analysed during the current study are available from the corresponding author on reasonable request.

\section{Competing interests}

The authors declare no competing interests. 


\section{Consortia}

\section{The Korean Organ Transplantation Registry Study Group}

Jin Min Kong6, Oh Jung Kwon? ${ }^{7}$, Deok Gie Kim ${ }^{8}$, Cheol-Woong Jung9, Yeong Hoon Kim $^{10}$, Joong Kyung Kim${ }^{11}$, Chan-Duck Kim ${ }^{12}$, Ji Won Min ${ }^{13}$, Sung Kwang Park ${ }^{14}$, Yeon Ho Park ${ }^{15}$, Park Jae Berm ${ }^{16}$, Jung Hwan Park ${ }^{17}$, Jong-Won Park ${ }^{18}$, Ho Sik Shin ${ }^{19}$, Hye Eun Yoon ${ }^{20}$, Kang Wook Lee ${ }^{21}$, Dong Ryeol Lee ${ }^{22}$, Dong Won Lee ${ }^{23}$, Sam Yeol Lee ${ }^{24}$, Sang-Ho Lee ${ }^{25}$, Su Hyung Lee ${ }^{26}$, Jung Jun Lee ${ }^{27}$, Lee Jung Pyo ${ }^{28}$, Jeong-Hoon Lee ${ }^{29}$, Jin Seok Jeon ${ }^{30}$, Heungman Jun ${ }^{31}$, KYUNGHWAN JEONG ${ }^{32}$, Ku Yong Chung ${ }^{33}$, Hong Rae $\mathrm{Cho}^{34}$, Ju Man Ki ${ }^{35}$, Dong-Wan Chae ${ }^{36}$, Soo Jin Na Choi ${ }^{37}$, Duck Jong Han ${ }^{38}$, Seungyeup Han $^{39}$, Kyu Ha Huh ${ }^{40}$

${ }^{6}$ Department of Nephrology, BHS Hanseo Hospital. ${ }^{7}$ Department of Surgery, College of Medicine, Han Yang University. ${ }^{8}$ Department of Surgery, Yonsei University Wonju College of Medicine, Wonju Severance Christian Hospital. ${ }^{9}$ Department of Transplantation and Vascular Surgery, Korea University Anam Hospital. ${ }^{10}$ Department of Internal Medicine, Inje University Busan Paik Hospital. ${ }^{11}$ Department of Internal Medicine, Bongseng Memorial Hospital. ${ }^{12}$ Department of Internal Medicine, School of Medicine, Kyungpook National University Hospital. ${ }^{13}$ Division of Nephrology, Department of Internal Medicine, Bucheon St. Mary's Hospital. ${ }^{14}$ Department of Internal Medicine, Chonbuk National University Medical School. ${ }^{15}$ Department of Surgery, Gil Medical Center, Gachon University College of Medicine. ${ }^{16}$ Department of Surgery, Samsung Medical Center, Sungkyunkwan University School of Medicine. ${ }^{17}$ Konkuk University School of Medicine, Department of Nephrology. ${ }^{15}$ Department of Nephrology, Yeungnam University Hospital. ${ }^{18}$ Kosin University College of 
Medicine, Department of Internal Medicine, Division of Nephrology. ${ }^{19}$ Department of Internal Medicine, Incheon St. Mary's Hospital. ${ }^{20}$ Department of Nephrology, Chungnam National University Hospital. ${ }^{21}$ Division of Nephrology, Department of Internal Medicine, Maryknoll Medical Center. ${ }^{22}$ Division of Nephrology, Department of Internal Medicine, Pusan National University School of Medicine. ${ }^{23}$ Department of Surgery, Kangdong Sacred Heart Hospital, Hallym University College of Medicine. ${ }^{24}$ Department of Nephrology, Kyung Hee University Hospital at Gangdong. ${ }^{25}$ Department of Surgery, Ajou University School of Medicine. ${ }^{26}$ Department of Surgery, CHA Bundang Medical Center. ${ }^{27}$ Department of Nephrology, SNU Boramae Medical Center. ${ }^{28}$ Department of Surgery, Myongji Hospital. 29 Department of Internal Medicine, Soonchunhyang University Seoul Hospital. 30 Department of Surgery, Inje University Ilsan Paik Hospital. ${ }^{31}$ Department of Internal Medicine, Kyung Hee University College of Medicine. ${ }^{32}$ Department of Surgery, Ewha Womans University Mokdong Hospital. ${ }^{33}$ Department of Surgery, Ulsan University Hospital. 34 Department of Surgery, Gangnam Severance Hospital, Yonsei University College of Medicine. ${ }^{35}$ Division of Nephrology, Seoul National University Bundang Hospital. ${ }^{36}$ Department of Surgery, Chonnam National University Medical School. ${ }^{37}$ Department of Surgery, Asan Medical Center. ${ }^{38}$ Department of Internal Medicine, Keimyung University School of Medicine. ${ }^{39}$ Department of Transplantation Surgery, Severance Hospital. 


\section{Figure legends}

\section{Figure 1}

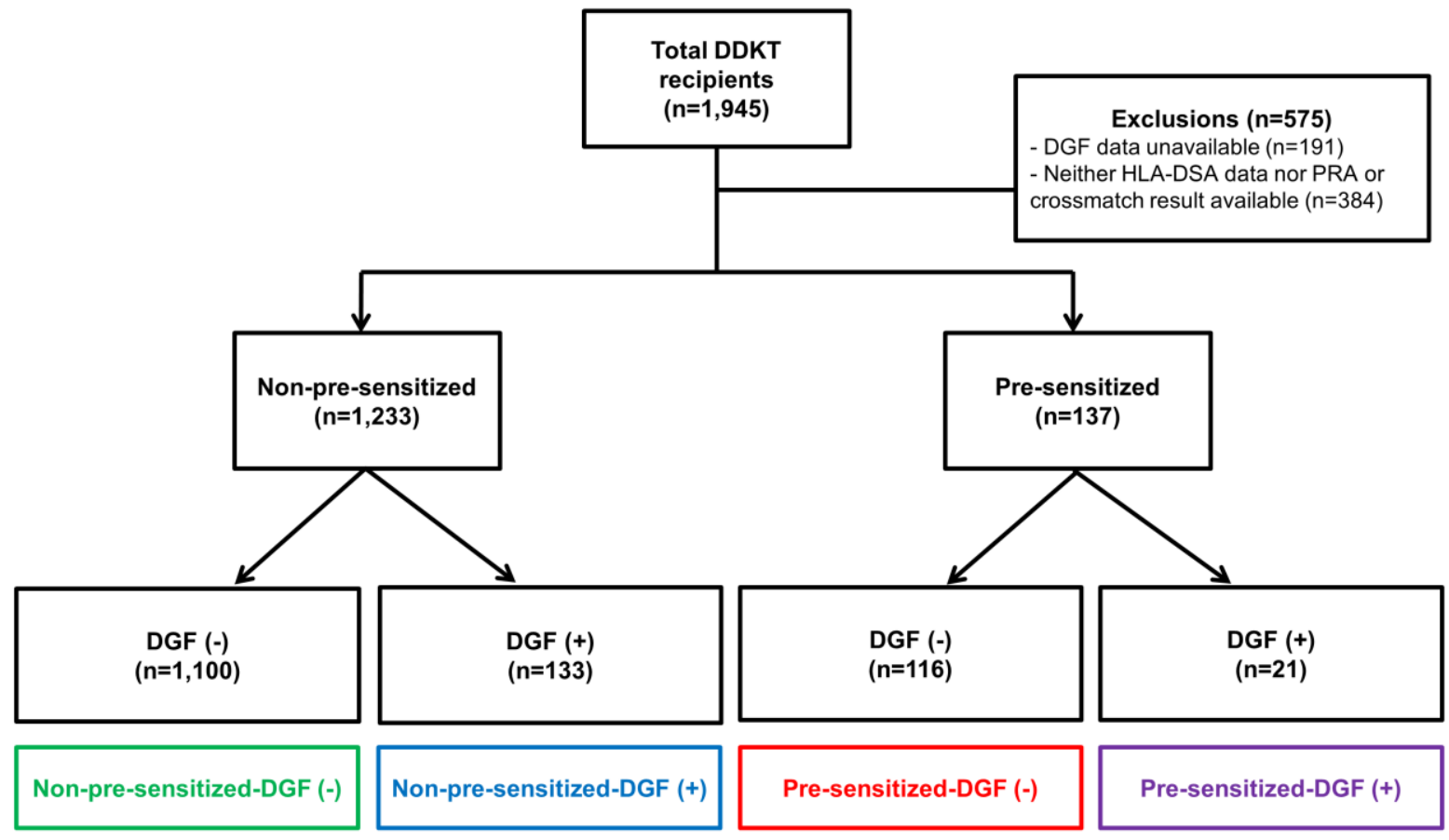

Figure 1. Distribution of the patient population according to DGF or pre-sensitization to

HLA. DGF, delayed graft function; HLA, human leukocyte antigen; DDKT, deceased donor kidney transplantation; DSA, donor-specific antibody. 


\section{Figure 2}

a.

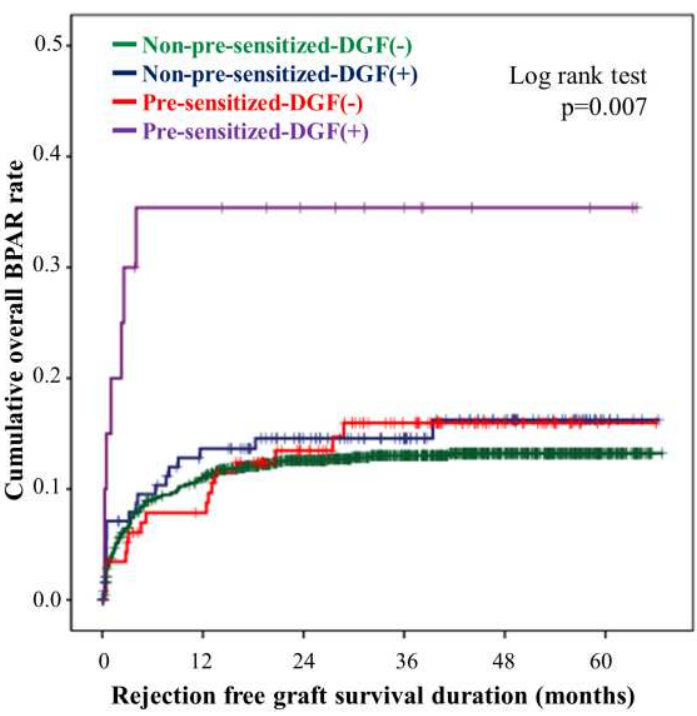

Number at risk

$\begin{array}{ccccccc}- & 1,100 & 941 & 742 & 511 & 276 & 71 \\ - & 133 & 103 & 83 & 63 & 39 & 6 \\ - & 116 & 102 & 75 & 55 & 23 & 2 \\ - & 21 & 12 & 9 & 6 & 3 & 2\end{array}$

b.

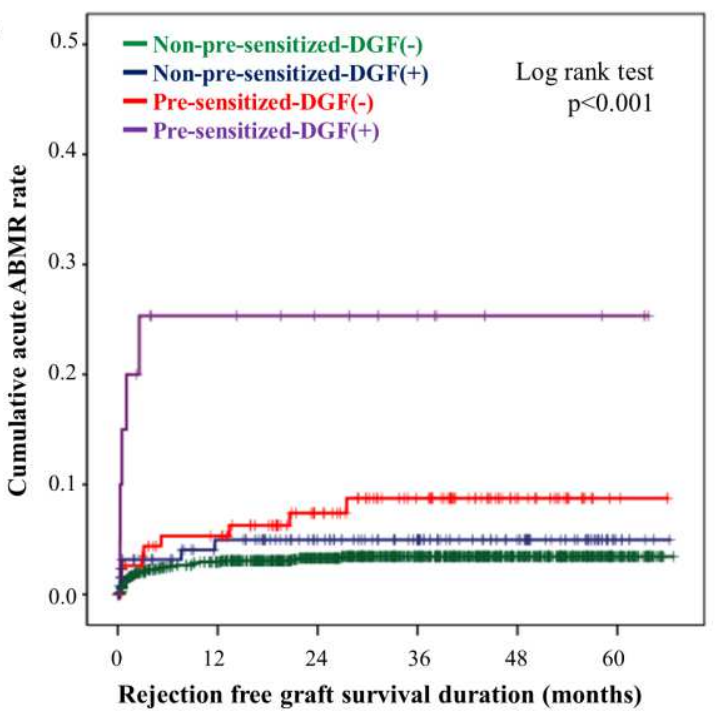

Number at risk

Figure 2. Kaplan-Meier estimates of withdrawal-censored cumulative (a) overall BPAR rate and (b) acute ABMR rate according to DGF and pre-sensitization status. The numbers below the figures denote the number of KTRs at risk in each subgroup. Note that both overall BPAR and acute ABMR rate increased in pre-sensitized-DGF(+) subgroup. DGF, delayed graft function; KTR, kidney transplantation recipient, BPAR, biopsy-proven allograft rejection, ABMR antibody-mediated rejection. 
Figure 3

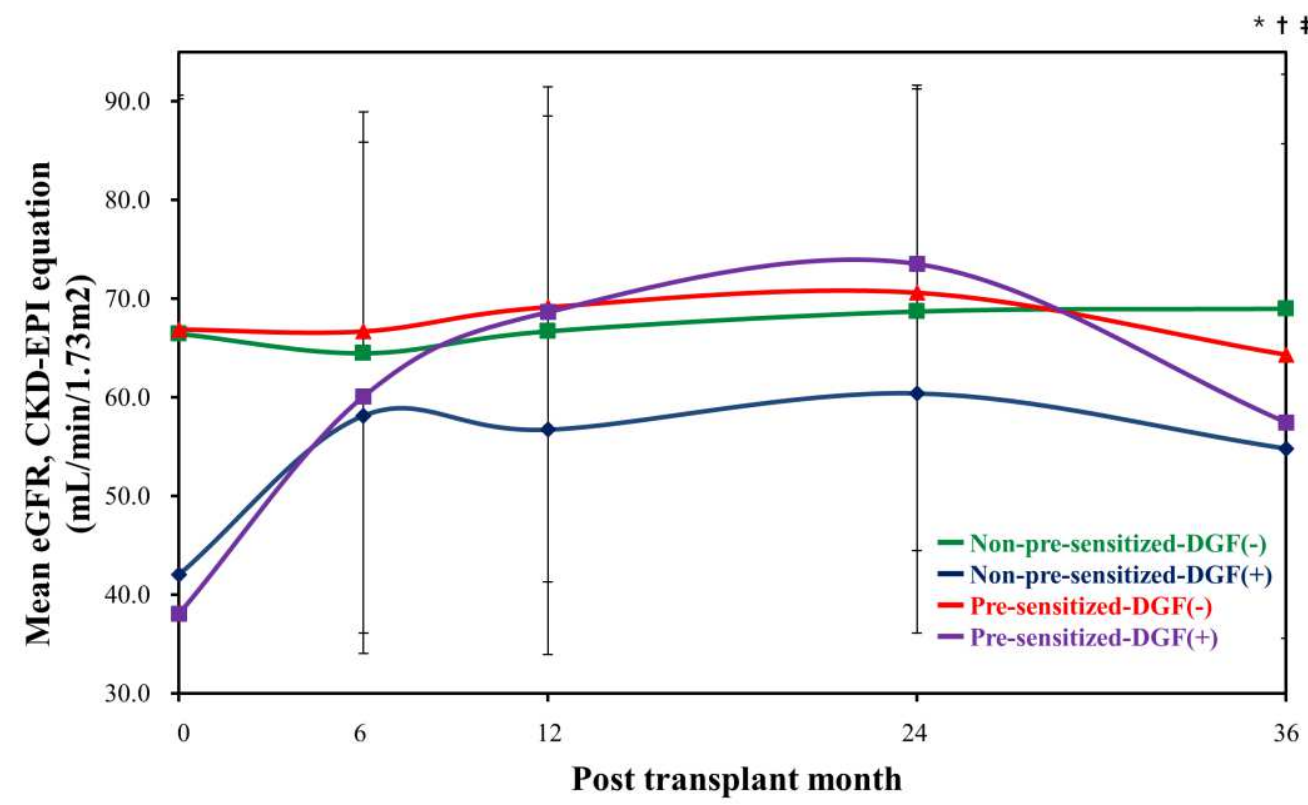

Number at risk

$\begin{array}{cccccc}- & 1,100 & 1,073 & 915 & 658 & 400 \\ - & 133 & 118 & 103 & 80 & 47 \\ - & 116 & 113 & 97 & 66 & 41 \\ - & 21 & 20 & 14 & 9 & 3\end{array}$

Figure 3. Comparison of the time-related changes in allograft function based on eGFR using CKD-EPI equation ( $\mathrm{mL} / \mathrm{min} / \mathbf{1 . 7 3 \mathrm { m } ^ { 2 }}$ ) according to DGF and pre-sensitization status. During 36 months, the non-pre-sensitized-DGF(+) subgroup showed the lowest allograft function compared with other subgroups. eGFR, estimated glomerular filtration rate; CKD-EPI, chronic kidney disease-epidemiology collaboration; DGF, delayed graft function.

${ }^{*} \mathrm{p}<0.001$ non-pre-sensitized-DGF(-) vs. non-pre-sensitized-DGF(+), ${ }^{\dagger} \mathrm{p}<0.001$ non-presensitized-DGF(+) vs. pre-sensitized-DGF(-), ${ }^{*} \mathrm{p}=0.034$ non-pre-sensitized-DGF(-) vs. presensitized-DGF(+). 
Figure 4
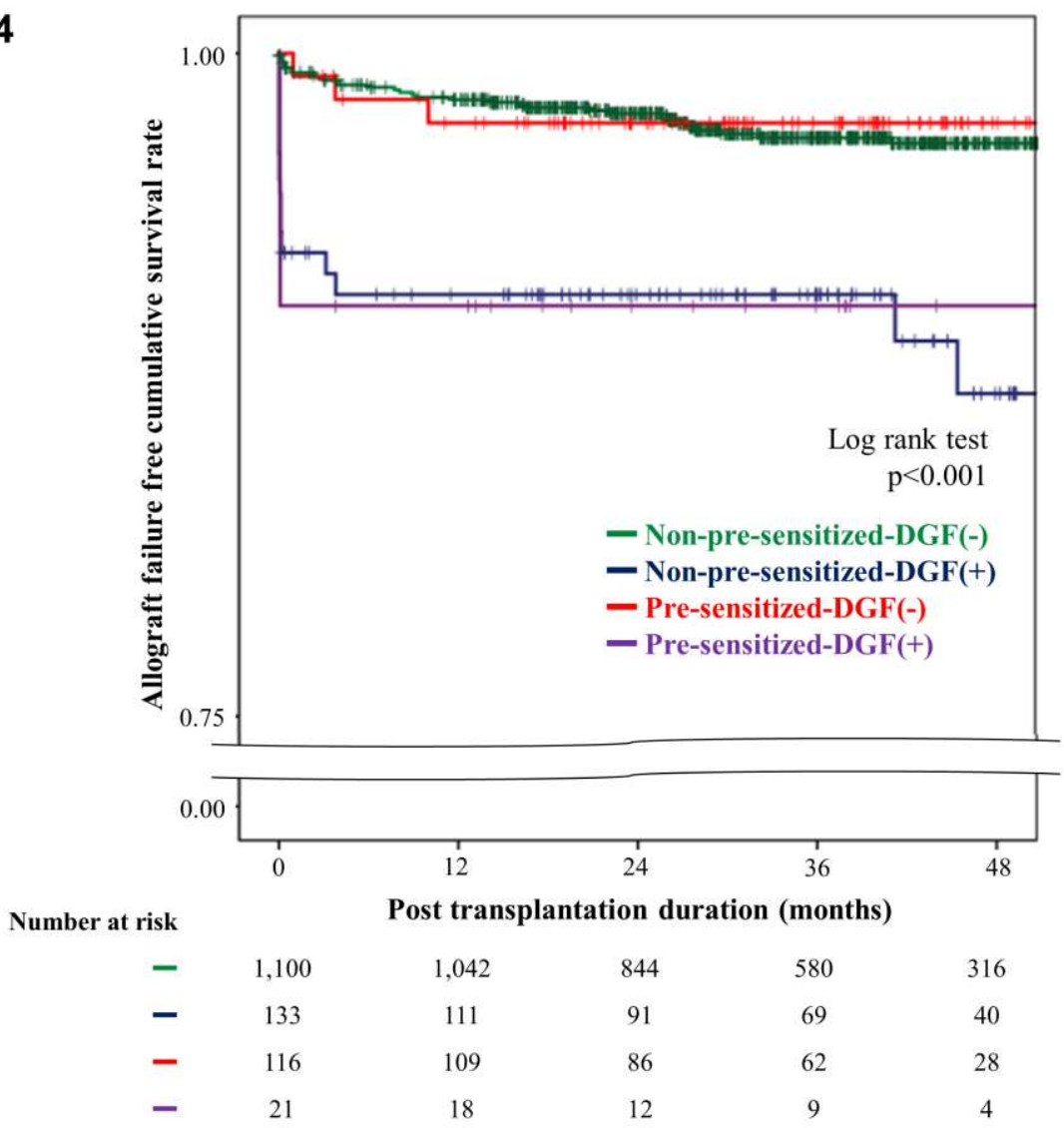

Figure 4. Kaplan-Meier estimates of death-censored allograft survival according to DGF and pre-sensitization status. The numbers below the figures denote the number of KTRs at risk in each subgroup. Note that the mortality rates were reduced in non-pre-sensitizedDGF(+) subgroup. DGF, delayed graft function; KTR, kidney transplantation recipient. 
Table 1. Comparison of clinical and laboratory parameters among the 4 subgroups according to DGF and pre-sensitization status.

\begin{tabular}{|c|c|c|c|c|c|}
\hline & \multicolumn{2}{|c|}{ Non-pre-sensitized $(\mathrm{n}=1,233)$} & \multicolumn{2}{|c|}{$\underline{\text { Pre-sensitized }(\mathrm{n}=137)}$} & \multirow{2}{*}{$p$-value } \\
\hline & $\operatorname{DGF}(-)(n=1,100)$ & $\operatorname{DGF}(+)(\mathrm{n}=133)$ & $\operatorname{DGF}(-)(n=116)$ & $\operatorname{DGF}(+)(\mathrm{n}=21)$ & \\
\hline \multicolumn{6}{|l|}{ Donors } \\
\hline Age (years) & $47.6 \pm 14.9$ & $48.3 \pm 14.9$ & $49.2 \pm 13.6$ & $47.0 \pm 12.9$ & 0.672 \\
\hline Male (n, \%) & $773(70.3 \%)$ & $95(71.4 \%)$ & $83(71.6 \%)$ & $12(57.1 \%)$ & 0.591 \\
\hline BMI $\left(\mathrm{kg} / \mathrm{m}^{2}\right)$ & $23.2 \pm 3.7$ & $23.5 \pm 3.9$ & $22.9 \pm 3.6$ & $23.0 \pm 4.8$ & 0.650 \\
\hline HTN (n, \%) & $261(23.7 \%)$ & $37(27.8 \%)$ & $21(18.1 \%)$ & $3(14.3 \%)$ & 0.194 \\
\hline $\mathrm{DM}(\mathrm{n}, \%)$ & $120(11.5 \%)$ & $13(9.9 \%)$ & $12(10.8 \%)$ & $1(5.0 \%)$ & 0.772 \\
\hline $\operatorname{DBD}(\mathrm{n}, \%)$ & $1059(96.3 \%)$ & $129(97.0 \%)$ & $112(96.6 \%)$ & $20(95.2 \%)$ & 0.967 \\
\hline $\operatorname{DCD}(\mathrm{n}, \%)$ & $41(3.7 \%)$ & $4(3.0 \%)$ & $43.4 \%)$ & $1(4.8 \%)$ & 0.967 \\
\hline eGFR (CKD-EPI) (ml/min/1.73m²) & $77.4(43.2-107.1)$ & $34.6(21.2-64.5)^{*}$ & $72.2(45.9-103.8)^{\dagger}$ & $47.8(27.2-98.8)$ & $<0.001$ \\
\hline Cold ischemic time (min) & $290.1 \pm 137.9$ & $323.4 \pm 136.5$ & $284.4 \pm 124.7$ & $322.9 \pm 142.2$ & 0.083 \\
\hline KDPI $(\%)$ & $66.0(44.0-84.0)$ & $71.0(49.5-88.0)$ & $64.0(51.0-81.0)$ & $67.0(49.5-82.5)$ & 0.262 \\
\hline \multicolumn{6}{|l|}{ Recipients } \\
\hline Age (years) & $51.3 \pm 10.6$ & $52.8 \pm 11.1$ & $51.1 \pm 9.8$ & $48.9 \pm 12.3$ & 0.283 \\
\hline Male (n, \%) & $680(61.8 \%)$ & $81(60.9 \%)$ & $38(32.8 \%)^{*}, \uparrow$ & $9(42.9 \%)$ & $<0.001$ \\
\hline
\end{tabular}




\begin{tabular}{|c|c|c|c|c|c|}
\hline BMI $\left(\mathrm{kg} / \mathrm{m}^{2}\right)$ & $23.0 \pm 3.3$ & $22.9 \pm 3.3$ & $22.0 \pm 2.9^{*}$ & $22.8 \pm 3.0$ & 0.022 \\
\hline HTN (n, \%) & $989(90.1 \%)$ & $121(91.0 \%)$ & $97(84.3 \%)$ & $21(100.0 \%)$ & 0.093 \\
\hline $\operatorname{DM}(\mathrm{n}, \%)$ & $314(28.5 \%)$ & $39(29.3 \%)$ & $17(14.7 \%)^{*}, \dagger$ & $4(19.0 \%)$ & 0.011 \\
\hline \multicolumn{6}{|l|}{ Dialysis modality (n, \%) } \\
\hline Hemodialysis & $859(78.1 \%)$ & $117(88.0 \%)^{*}$ & $97(83.6 \%)$ & $20(95.2 \%)$ & 0.009 \\
\hline Peritoneal dialysis & $241(21.9 \%)$ & $16(12.0 \%)^{*}$ & $19(16.4 \%)$ & $1(4.8 \%)$ & 0.009 \\
\hline Dialysis duration (months) & $84.5(53.6-113.4)$ & $90.4(50.3-113.9)$ & $103.7(68.5-136.0)^{* \dagger}$ & $130.5(108.9-161.2)^{*}, \dagger$ & $<0.001$ \\
\hline Previous KT history (n, \%) & $93(8.5 \%)$ & $15(11.3 \%)$ & $31(36.7 \%)^{* \dagger}$ & $4(19.0 \%)$ & $<0.001$ \\
\hline Mismatch number (n) & $3.4 \pm 1.8$ & $3.6 \pm 1.6$ & $3.8 \pm 1.3^{*}$ & $3.6 \pm 1.6$ & 0.022 \\
\hline \multicolumn{6}{|l|}{ Induction therapy (n, \%) } \\
\hline ATG & $311(28.3 \%)$ & $59(44.4 \%)^{*}$ & $69(59.5 \%)^{*}, \dagger$ & $13(61.9 \%)^{*}$ & $<0.001$ \\
\hline Basiliximab & $804(73.2 \%)$ & $88(66.2 \%)$ & $59(50.9 \%)^{*}, \dagger$ & $15(71.4 \%)$ & $<0.001$ \\
\hline \multicolumn{6}{|c|}{ Main immunosuppressant (n, \%) } \\
\hline Tacrolimus & $1077(97.9 \%)$ & $130(97.7 \%)$ & $115(99.1 \%)$ & $19(90.5 \%)$ & 0.091 \\
\hline Cyclosporin & $17(1.5 \%)$ & $0(0.0 \%)$ & $0(0.0 \%)$ & $1(4.8 \%)$ & 0.127 \\
\hline Sirolimus & $8(0.7 \%)$ & $4(3.0 \%)^{*}$ & $1(0.9 \%)$ & $1(4.8 \%)$ & 0.029 \\
\hline PRA $>50 \%$ & $327(29.7 \%)$ & $40(30.1 \%)$ & $95(81.9 \%)^{*, \dagger}$ & $16(76.2 \%)^{*}, \dagger$ & $<0.001$ \\
\hline
\end{tabular}


Continuous variables are shown as mean \pm standard deviation or median with interquartile range. Categorical variables are shown as number (proportions). DGF, delayed graft function; BMI, body mass index; HTN, hypertension; DM, diabetes mellitus; DBD, donor after brain death; DCD, donor after cardiac death; eGFR, estimated glomerular filtration; CKD-EPI, chronic kidney disease-epidemiology collaboration; KDPI, kidney donor profile index; KT, kidney transplantation; ATG, anti-thymocyte globulin; PRA, panel reactive antibody; DSA, donor-specific antibody.

${ }^{*} p<0.05$ compared with non-pre-sensitized-DGF(-) subgroup, ${ }^{\dagger} p<0.05$ compared with non-pre-sensitized- DGF(+) subgroup 
Table 2. Comparison of rejection-related outcomes among the 4 subgroups according to DGF and pre-sensitization status.

\begin{tabular}{|c|c|c|c|c|c|}
\hline & \multicolumn{2}{|c|}{ Non-pre-sensitized $(\mathrm{n}=1,233)$} & \multicolumn{2}{|c|}{$\underline{\text { Pre-sensitized }(n=137)}$} & \multirow{2}{*}{$\begin{array}{c}p \text { - } \\
\text { value }\end{array}$} \\
\hline & $\begin{array}{c}\text { DGF }(-) \\
(\mathrm{n}=1,100)\end{array}$ & $\begin{array}{l}\text { DGF (+) } \\
(\mathrm{n}=133)\end{array}$ & $\begin{array}{l}\text { DGF (-) } \\
(\mathrm{n}=116)\end{array}$ & $\begin{array}{l}\operatorname{DGF}(+) \\
(n=21)\end{array}$ & \\
\hline Overall BPAR (n, \%) & $139(12.6 \%)$ & $19(14.3 \%)$ & $17(14.7 \%)$ & $7(33.3 \%)^{*}$ & 0.045 \\
\hline Acute ABMR (n, \%) & $35(3.2 \%)$ & $6(4.5 \%)$ & $9(7.8 \%)^{*}$ & $5(23.8 \%)^{*}, \uparrow, \dagger$ & $<0.001$ \\
\hline Acute TCMR (n, \%) & $107(9.7 \%)$ & $11(8.3 \%)$ & $9(7.8 \%)$ & $4(19.0 \%)$ & 0.405 \\
\hline Chronic active ABMR (n, \%) & $3(0.3 \%)$ & $2(1.5 \%)$ & $4(4.3 \%)^{*}$ & $1(4.8 \%)$ & $<0.001$ \\
\hline Chronic active TCMR (n, \%) & $10(0.9 \%)$ & $3(2.3 \%)$ & $1(0.9 \%)$ & $1(4.8 \%)$ & 0.198 \\
\hline $\begin{array}{l}\text { Repeated acute rejection } \\
\text { within } 1 \text { year }(n, \%)\end{array}$ & $41(3.7 \%)$ & $5(3.8 \%)$ & $5(4.3 \%)$ & $3(14.3 \%)$ & 0.106 \\
\hline
\end{tabular}

Categorical variables are shown as number (proportions). DGF, delayed graft function; BPAR, biopsy-proven allograft rejection; ABMR, antibody-mediated rejection, TCMR, T-cell mediated rejection.

${ }^{*} p<0.05$ compared with non-pre-sensitized-DGF(-) subgroup, ${ }^{\dagger} p<0.05$ compared with non-pre-sensitizedDGF(+) subgroup, ${ }^{\star} p<0.05$ compared with pre-sensitized-DGF(-) subgroup. 
Table 3. Multivariable Cox regression for independent predictors of (a) overall BPAR and (b) acute ABMR.

\begin{tabular}{|c|c|c|c|c|c|}
\hline (a) & $\begin{array}{c}\text { Unadjusted HR } \\
\quad(95 \% \mathrm{CI})\end{array}$ & $p$-value & $\begin{array}{c}\text { Adjusted HR } \\
(95 \% \mathrm{CI})\end{array}$ & $p$-value & $\begin{array}{l}p \text {-value for } \\
\text { interaction }\end{array}$ \\
\hline Donor age & $1.014(1.003-1.024)$ & 0.012 & & & \\
\hline Cold ischemic time & $0.998(0.997-1.000)$ & 0.016 & $0.998(0.997-1.000)$ & 0.035 & \\
\hline KDPI & $1.006(1.000-1.012)$ & 0.049 & & & \\
\hline Recipient BMI & $1.055(1.012-1.099)$ & 0.011 & & & \\
\hline Prior KT history & $1.197(0.766-1.869)$ & 0.429 & & & \\
\hline Mismatch number & $1.131(1.036-1.235)$ & 0.006 & $1.094(0.984-1.217)$ & 0.097 & \\
\hline $\mathrm{PRA}>50 \%$ & $1.074(0.793-1.455)$ & 0.644 & & & \\
\hline Pre-sensitization \& DGF & & & & & 0.006 \\
\hline Non-pre-sensitized/DGF (-) & Reference & & Reference & & \\
\hline Non-pre-sensitized/DGF (+) & $1.210(0.749-1.954)$ & 0.437 & $1.309(0.747-2.294)$ & 0.347 & \\
\hline Pre-sensitized/DGF (-) & $1.150(0.695-1.902)$ & 0.587 & $1.057(0.604-1.848)$ & 0.847 & \\
\hline Pre-sensitized/DGF (+) & $3.489(1.633-7.456)$ & 0.001 & $3.253(1.423-7.434)$ & 0.005 & \\
\hline
\end{tabular}

\begin{tabular}{|c|c|c|c|c|c|}
\hline (b) & $\begin{array}{l}\text { Unadjusted HR } \\
\qquad(95 \% \mathrm{CI})\end{array}$ & $p$-value & $\begin{array}{c}\text { Adjusted HR } \\
(95 \% \mathrm{CI})\end{array}$ & $\begin{array}{c}p- \\
\text { value }\end{array}$ & $\begin{array}{l}p \text {-value for } \\
\text { interaction }\end{array}$ \\
\hline Donor age & $0.998(0.980-1.016)$ & 0.835 & & & \\
\hline Cold ischemic time & $0.999(0.997-1.002)$ & 0.601 & & & \\
\hline KDPI & $0.997(0.987-1.008)$ & 0.588 & & & \\
\hline Recipient BMI & $1.002(0.924-1.087)$ & 0.959 & & & \\
\hline Prior KT history & $2.176(1.124-4.213)$ & 0.021 & $2.493(1.201-5.177)$ & 0.014 & \\
\hline Mismatch number & $1.020(0.875-1.188)$ & 0.803 & & & \\
\hline $\mathrm{PRA}>50 \%$ & $1.862(1.097-3.159)$ & 0.021 & & & \\
\hline Pre-sensitization \& DGF & & & & & $<0.001$ \\
\hline Non-pre-sensitized/DGF (-) & Reference & & Reference & & \\
\hline
\end{tabular}




\begin{tabular}{l|cccc} 
Non-pre-sensitized/DGF (+) & $1.518(0.638-3.608)$ & 0.345 & $1.712(0.657-4.459)$ & 0.271 \\
Pre-sensitized/DGF (-) & $2.423(1.165-5.041)$ & 0.018 & $1.974(0.872-4.470)$ & 0.103 \\
Pre-sensitized/DGF (+) & $9.525(3.730-24.326)$ & $<0.001$ & $7.589(2.644-21.777)$ & $<0.001$
\end{tabular}

1,027 recipients were included in multivariable Cox regression model. Multivariable regression model was adjusted with parameters showing significant differences in baseline characteristics or known to affect allograft rejection. Parameters were as follows: donor factors (age, cold ischemic time, KDPI), Recipient factors (BMI, prior KT history, mismatch number, PRA>50\%).

HR, hazard ratio; BPAR, biopsy-proven allograft rejection; ABMR, antibody-mediated rejection; DGF, delayed graft function; CI, confidence interval; BMI, body mass index; KDPI, kidney donor profile index; KT, kidney transplantation; PRA, panel reactive antibody. 
Table 4. (a) Causes of death and (b) clinical outcomes among the 4 subgroups according to DGF and pre-sensitization status.

\begin{tabular}{|c|c|c|c|c|c|}
\hline \multirow[b]{2}{*}{ (a) } & \multicolumn{2}{|c|}{ Non-pre-sensitized $(\mathrm{n}=1,233)$} & \multicolumn{2}{|c|}{ Pre-sensitized $(\mathrm{n}=137)$} & \multirow{2}{*}{$\begin{array}{c}p- \\
\text { value }\end{array}$} \\
\hline & $\begin{array}{c}\text { DGF }(-) \\
(\mathrm{n}=1,100)\end{array}$ & $\begin{array}{l}\mathrm{DGF}(+) \\
(\mathrm{n}=133)\end{array}$ & $\begin{array}{c}\operatorname{DGF}(-) \\
(\mathrm{n}=116)\end{array}$ & $\begin{array}{l}\operatorname{DGF}(+) \\
(\mathrm{n}=21)\end{array}$ & \\
\hline Total $(\mathrm{n}, \%)$ & $37(3.4 \%)$ & $16(12.0 \%)$ & $4(3.4 \%)$ & $0(0.0 \%)$ & 0.001 \\
\hline Cardiovascular disease $(\mathrm{n}, \%)$ & $7(18.9 \%)$ & $1(6.4 \%)$ & $1(25.0 \%)$ & $0(0.0 \%)$ & \\
\hline Infection (n, \%) & $20(54.1 \%)$ & $5(31.3 \%)$ & $2(50.0 \%)$ & $0(0.0 \%)$ & \\
\hline Malignancy (n, \%) & $1(2.7 \%)$ & $2(12.5 \%)$ & $1(25.0 \%)$ & $0(0.0 \%)$ & \\
\hline Others (n, \%) & $8(21.6 \%)$ & $4(25.0 \%)$ & $0(0.0 \%)$ & $0(0.0 \%)$ & \\
\hline Unknown (n, \%) & $1(2.7 \%)$ & $4(25.0 \%)$ & $0(0.0 \%)$ & $0(0.0 \%)$ & \\
\hline
\end{tabular}

(b)

$\underline{\text { Non-pre-sensitized ( } \mathrm{n}=1,233)}$

$\underline{\text { Pre-sensitized }(\mathrm{n}=137)}$

\begin{tabular}{|c|c|c|c|c|c|}
\hline \multirow[b]{2}{*}{ (b) } & \multicolumn{2}{|c|}{ Non-pre-sensitized $(\mathrm{n}=1,233)$} & \multicolumn{2}{|c|}{ Pre-sensitized $(\mathrm{n}=137)$} & \multirow[b]{2}{*}{$\begin{array}{c}p- \\
\text { value }\end{array}$} \\
\hline & $\begin{array}{c}\operatorname{DGF}(-) \\
(\mathrm{n}=1,100)\end{array}$ & $\begin{array}{l}\text { DGF (+) } \\
(\mathrm{n}=133)\end{array}$ & $\begin{array}{l}\text { DGF (-) } \\
(\mathrm{n}=116)\end{array}$ & $\begin{array}{l}\operatorname{DGF}(+) \\
(\mathrm{n}=21)\end{array}$ & \\
\hline BKVAN (n, \%) & $32(2.9 \%)$ & $4(3.0 \%)$ & $5(4.3 \%)$ & $0(0.0 \%)$ & 0.713 \\
\hline Cardiovascular disease $(\mathrm{n}, \%)$ & $142(12.9 \%)$ & $25(18.8 \%)$ & $14(12.1 \%)$ & $0(0.0 \%)^{\dagger}$ & 0.071 \\
\hline Cerebrovascular disease $(\mathrm{n}, \%)$ & $10(0.9 \%)$ & $1(0.8 \%)$ & $0(0.0 \%)$ & $0(0.0 \%)$ & 0.737 \\
\hline \multicolumn{6}{|l|}{ Infection } \\
\hline Overall (n, \%) & $277(33.1 \%)$ & $37(37.4 \%)$ & $37(42.0 \%)$ & $4(28.6 \%)$ & 0.316 \\
\hline CMV infection (n, \%) & $51(6.1 \%)$ & $4(4.0 \%)$ & $1(1.1 \%)$ & $0(0.0 \%)$ & 0.167 \\
\hline PJP infection (n, \%) & $5(0.6 \%)$ & $1(1.0 \%)$ & $1(1.1 \%)$ & $1(7.1 \%)^{*}$ & 0.046 \\
\hline Malignancy (n, \%) & $77(7.0 \%)$ & $11(8.3 \%)$ & $9(7.8 \%)$ & $2(9.5 \%)$ & 0.916 \\
\hline
\end{tabular}

Continuous variables are shown as mean \pm standard deviation or median with interquartile range. Categorical variables are shown as number (proportions). DGF, delayed graft function; CNI, calcineurin inhibitor; BKVAN, BK virus associated nephropathy; CMV, cytomegalovirus; PJP, Pneumocystis jirovecii pneumonia.

Others: liver disease, cerebral infarction, acute CNI toxicity, gastrointestinal bleeding, intestinal obstruction, acute rejection etc.

${ }^{*} p<0.05$ compared with non-pre-sensitized-DGF(-) subgroup, ${ }^{\dagger} p<0.05$ compared with non-pre-sensitizedDGF(+) subgroup. 


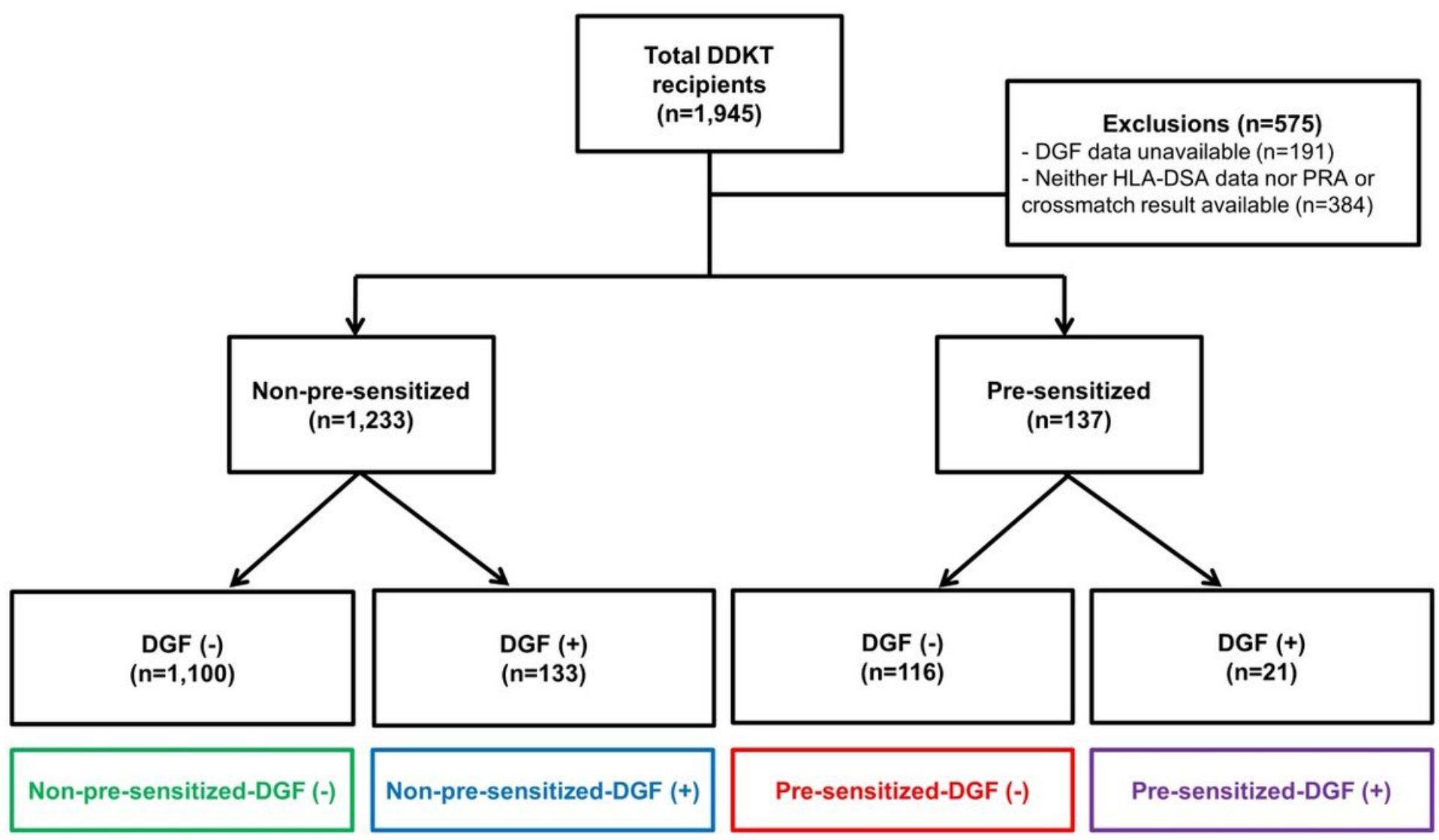

Figure 1

Distribution of the patient population according to DGF or pre sensitization to HLA. DGF, delayed graft function; HLA, human leukocyte antigen; DDKT, deceased donor kidney transplantation; DSA, donor specific antibody. 
a.

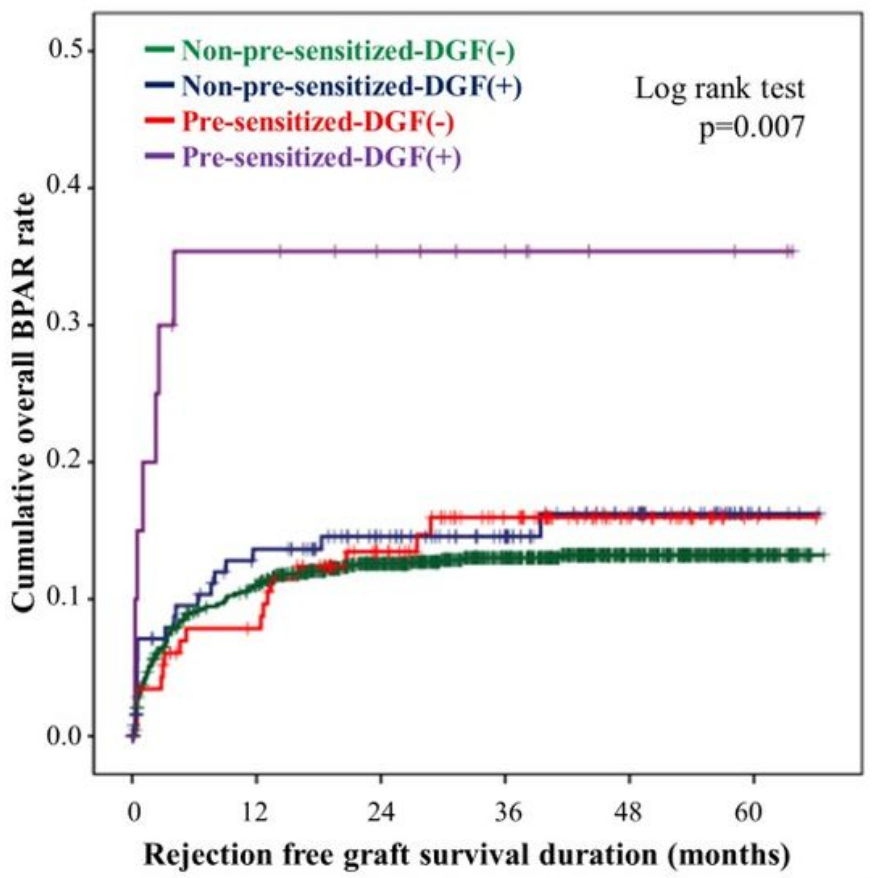

b.

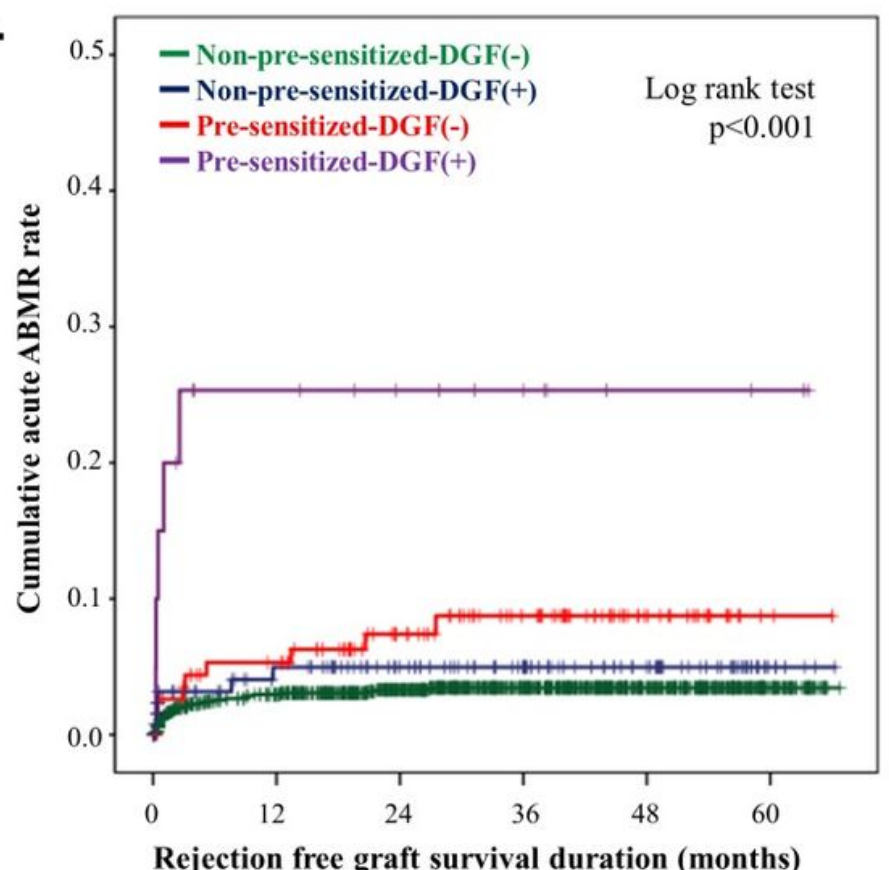

Number at risk
Number at risk

$\begin{array}{ccccccc}- & 1,100 & 941 & 742 & 511 & 276 & 71 \\ - & 133 & 103 & 83 & 63 & 39 & 6 \\ - & 116 & 102 & 75 & 55 & 23 & 2 \\ - & 21 & 12 & 9 & 6 & 3 & 2\end{array}$

$\begin{array}{ccccccc}- & 1,100 & 941 & 742 & 511 & 276 & 71 \\ - & 133 & 103 & 83 & 63 & 39 & 6 \\ - & 116 & 102 & 75 & 55 & 23 & 2 \\ - & 21 & 12 & 9 & 6 & 3 & 2\end{array}$

\section{Figure 2}

Kaplan Meier estimates of withdrawal censored cumulative (a) overall BPAR rate and (b) acute ABMR rate according to DGF and pre sensitization status. The numbers below the figures denote the number of KTRs at risk in each subgroup. Note that both overall BPA R and acute ABMR rate increased in pre sensitized DGF(+) subgroup. DGF, delayed graft function; KTR, kidney transplantation recipient, BPAR, biopsy proven allograft rejection, ABMR antibody mediated rejection. 


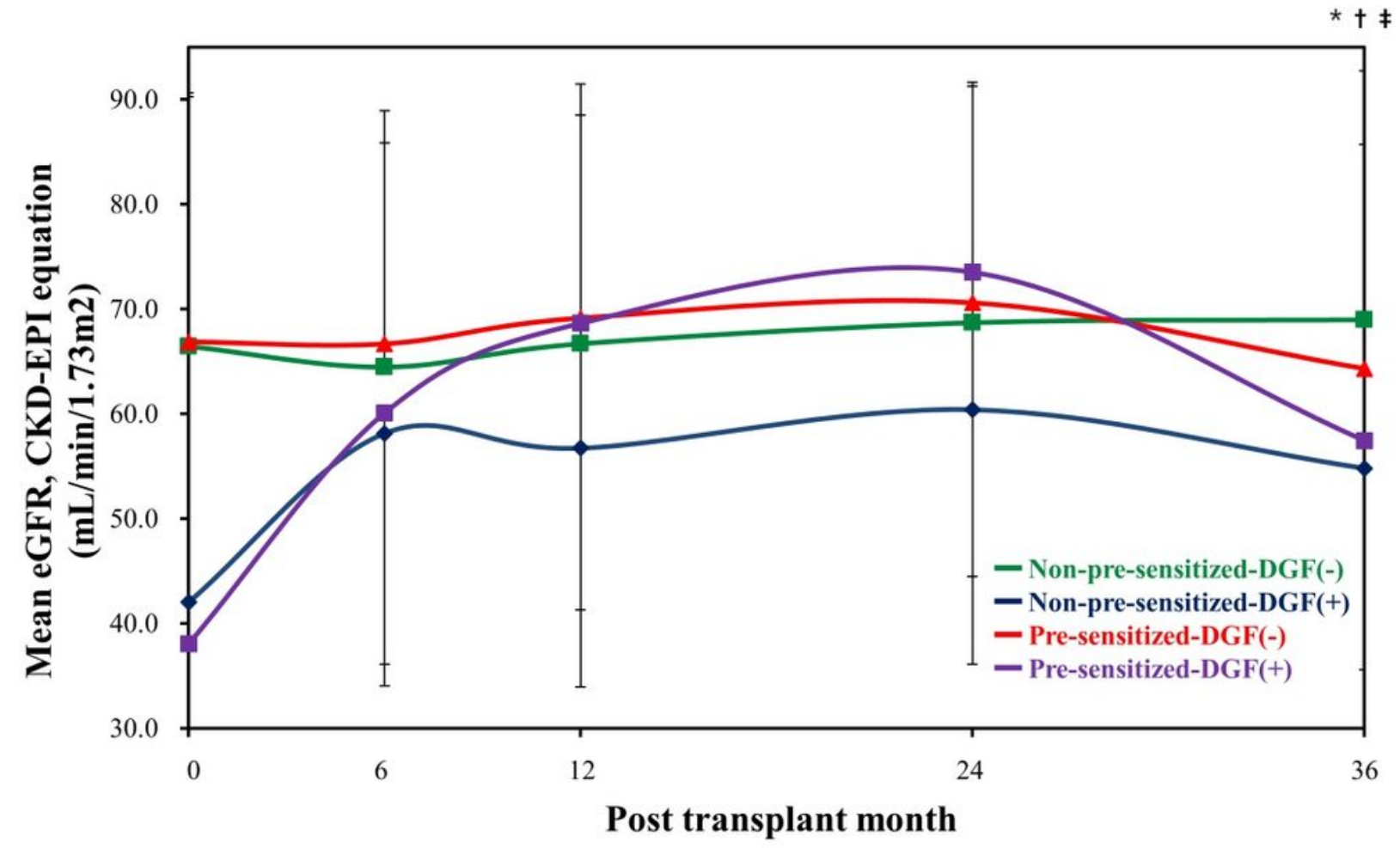

Number at risk

$\begin{array}{cccc}- & 1,100 & 1,073 & 915 \\ - & 133 & 118 & 103 \\ - & 116 & 113 & 97 \\ - & 21 & 20 & 14\end{array}$

\section{Figure 3}

Comparison of the time-related changes in allograft function based on eGFR using CKD-EPI equation $(\mathrm{mL} / \mathrm{min} / 1.73 \mathrm{~m} 2)$ according to DGF and pre-sensitization status. During 36 months, the non-presensitized-DGF(+) subgroup showed the lowest allograft function compared with other subgroups. eGFR, estimated glomerular filtration rate; CKD-EPI, chronic kidney disease-epidemiology collaboration; DGF, delayed graft function. * $p<0.001$ non-pre-sensitized-DGF(-) vs. non-pre-sensitized-DGF $(+),+p<0.001$ nonpre-sensitized-DGF(+) vs. pre-sensitized-DGF(-), $\neq p=0.034$ non-pre-sensitized-DGF(-) vs. pre-sensitized$\operatorname{DGF}(+)$. 

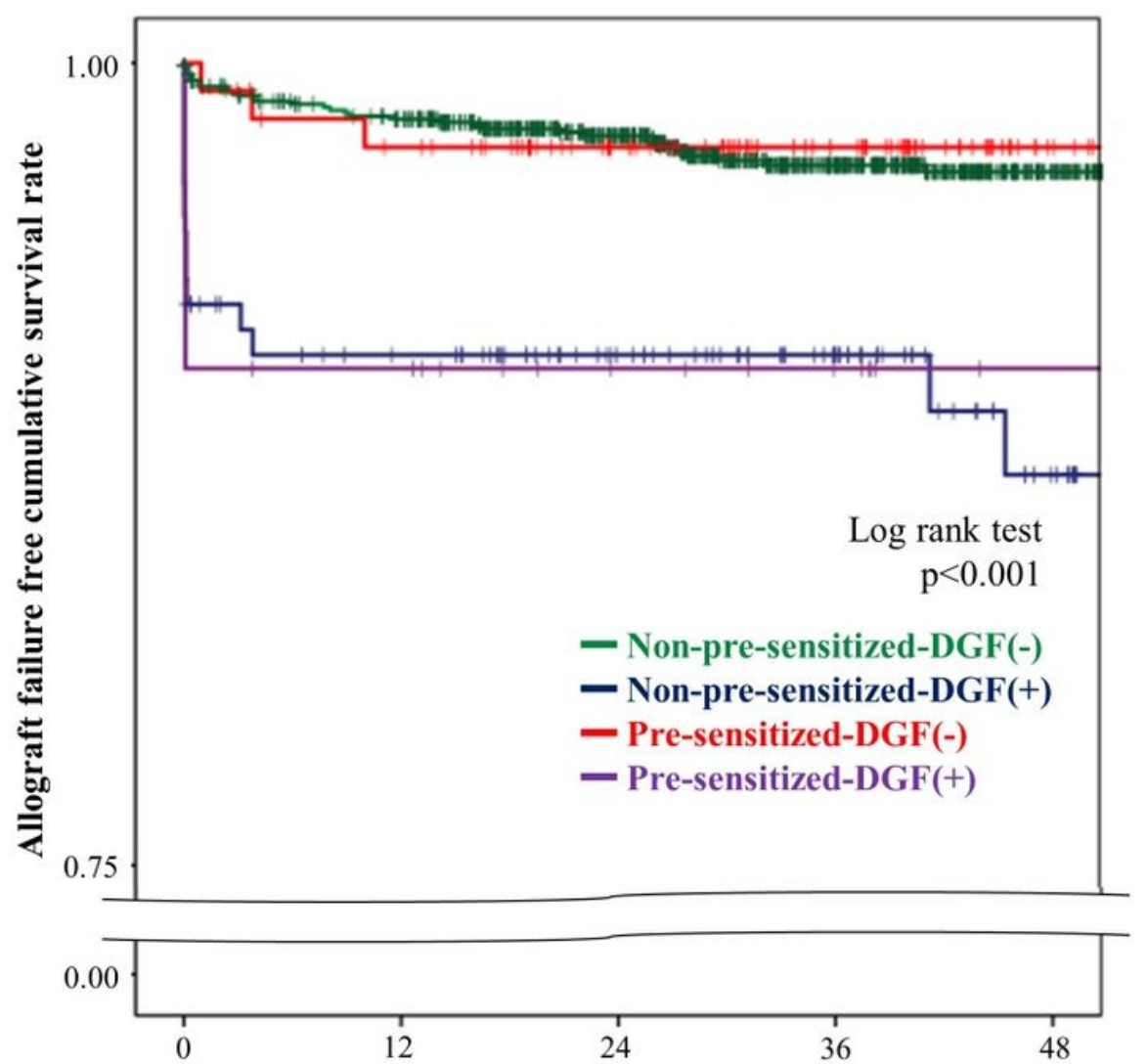

Number at risk

Post transplantation duration (months)

$\begin{array}{cccccc}- & 1,100 & 1,042 & 844 & 580 & 316 \\ - & 133 & 111 & 91 & 69 & 40 \\ - & 116 & 109 & 86 & 62 & 28 \\ - & 21 & 18 & 12 & 9 & 4\end{array}$

\section{Figure 4}

Kaplan Meier estimates of death censored allograft survival according to DGF and pre sensitization status. The numbers below the figures denote the number of KTRs at risk in ea ch subgroup. Note that the mortality rates were reduced in non pre sensitized DGF(+) subgroup . DGF, delayed graft function; KTR, kidney transplantation

\section{Supplementary Files}

This is a list of supplementary files associated with this preprint. Click to download.

- LeeetaIDGFandpresensitizationKOTRYSciRsuppl.doc 